\title{
Alterações induzidas pela dieta com diferentes concentrações de amido resistente no metabolismo de carboidratos e de lipídeos, em ratos Wistar
}

\section{Changes induced by diet with different concentrations of resistant starch in the metabolism of} carbohydrates and lipids in Wistar rats

Alteraciones inducidas por la dieta con diferentes concentraciones de almidón resistente en el metabolismo de carbohidratos y lípidos, en ratas Wistar

Recebido: 24/05/2021 | Revisado: 01/06/2021 | Aceito: 02/06/2021 | Publicado: 18/06/2021

\author{
Armindo Antonio Alves \\ ORCID: https://orcid.org/0000-0003-3091-4818 \\ Centro Universitário Herminio Ometto de Araras, Brasil \\ E-mail: alvesaa@uol.com.br \\ Acácio Antônio Pigoso \\ ORCID: https://orcid.org/0000-0002-9415-5278 \\ Centro Universitário Herminio Ometto de Araras, Brasil \\ E-mail: acaciopigoso@fho.edu.br \\ Yoon Kil Chang \\ ORCID: https://orcid.org/0000-0002-5897-1681 \\ Universidade Estadual de Campinas, Brasil \\ E-mail: yoonkilch@gmail.com \\ Bruna Lago Tagliapietra \\ ORCID: https://orcid.org/0000-0003-3041-4768 \\ Universidade Estadual de Campinas, Brasil \\ E-mail: bruna_tagliapietra@hotmail.com \\ Marcio Schmiele \\ ORCID: https://orcid.org/0000-0001-8830-1710 \\ Universidade Federal dos Vales do Jequitinhonha e Mucuri, Brasil \\ E-mail: marcio.sc@ict.ufvjm.edu.br \\ Pedro Henrique Campelo \\ ORCID: https://orcid.org/0000-0002-5137-0162 \\ Universidade Federal do Amazonas, Brasil \\ E-mail: pedrocampelo@ufam.edu.br \\ Maria Teresa Pedrosa Silva Clerici \\ ORCID: https://orcid.org/0000-0002-8445-336X \\ Universidade Estadual de Campinas, Brasil \\ E-mail: mclerici@unicamp.br
}

\begin{abstract}
Resumo
Este trabalho teve como objetivo avaliar o efeito da substituição do amido pré-gelatinizado por amido resistente no metabolismo de lipídeos e de carboidratos em ratos machos Wistar. 20 ratos machos foram divididos em dois grupos, um grupo recebeu uma dieta com 9,5\% (DAP) e o outro grupo com 18,1\% (DAR) de amido resistente durante 22 dias. Foram analisados peso, consumo de ração e água, e quantificadas as concentrações de colesterol e de triglicerídeos no soro e no músculo, e de glicogênio hepático e muscular. Os dados foram analisados por teste de Tukey $(\mathrm{p}>0,05)$ e gráficos box-plot. Não houve diferenças significativas na ingestão de ração e água, mas os animais do grupo DAP

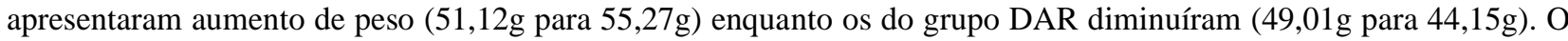
grupo DAR apresentou diminuição de triglicérideos, colesterol total no soro (de 125,761 mg/dL para 104,874 mg/dL) e do glicogênio muscular, mas aumento de triglicerídeos no músculo gastrocnêmio em comparação com o grupo DAP (de $0,160 \mathrm{mg} / \mathrm{dL}$ para $0,259 \mathrm{mg} / \mathrm{dL}$ ). Estes resultados apontam para a diminuição na velocidade de absorção da glicose no grupo de animais com dieta com maior quantidade de amido resistente, que pode ter induzido diminuição nos processos de síntese de triglicérideos e de colesterol levando ao aumento do catabolismo destes substratos pelo organismo, e adaptação plástica dos músculos para utilização de ácidos graxos no seu metabolismo oxidativo.
\end{abstract}

Palavras-chave: Amido pré-gelatinizado; Dietas experimentais; Triglicerídeos; Glicogênio; Colesterol.

\section{Abstract}

This study aimed to evaluate the effect of replacing pregelatinized starch with resistant starch on the metabolism of lipids and carbohydrates in male Wistar rats. 20 male rats were divided into two groups, which received a diet with 9.5\% (DAP) and 18.1\% (DAR) of resistant starch for 22 days. Weight, feed, and water consumption were analyzed and the concentrations of cholesterol and triglycerides in serum and muscle and hepatic and muscle glycogen were quantified. The data were analyzed by Tukey's test $(\mathrm{p}>0.05)$ and box-plot graphs. There were no significant 
differences in feed and water intake, but the animals in the DAP group showed an increase in weight $(51.12 \mathrm{~g}$ to $55.27 \mathrm{~g}$ ) while those in the DAR group decreased (49.01g to $44.15 \mathrm{~g}$ ). The DAR group showed a decrease in triglycerides, total serum cholesterol (from $125.761 \mathrm{mg} / \mathrm{dL}$ to $104.874 \mathrm{mg} / \mathrm{dL}$ ), and muscle glycogen, but an increase in triglycerides in the gastrocnemius muscle compared to the DAP group (from $0.160 \mathrm{mg} / \mathrm{dL}$ to $0.259 \mathrm{mg} / \mathrm{dL}$ ). These results point to a decrease in the glucose absorption speed in the group of animals with a diet with a higher amount of resistant starch, which may have induced a decrease in the processes of synthesis of triglycerides and cholesterol leading to an increase in catabolism of these substrates by the body, plastic adaptation of muscles for the use of fatty acids in their oxidative metabolism.

Keywords: Pregelatinized starch; Experimental diets; Triglycerides; Glycogen; Cholesterol.

\section{Resumen}

Este estudio tuvo como objetivo evaluar el efecto de la sustitución del almidón pregelatinizado por almidón resistente sobre el metabolismo de lípidos y carbohidratos en ratas Wistar macho. Se dividieron 20 ratas macho en dos grupos, los cuales recibieron una dieta con 9.5\% (DAP) y 18.1\% (DAR) de almidón resistente durante 22 días. Se analizaron el peso, consumo de alimento y agua, y se cuantificaron las concentraciones de colesterol y triglicéridos en el suero y músculo, y el glucógeno hepático y muscular. Los datos fueron analizados mediante la prueba de Tukey ( $\mathrm{p}>0.05)$ y gráficos de diagrama de cajas. No hubo diferencias significativas en la ingesta de alimento y agua, sin embargo, los animales del grupo DAP mostraron un aumento de peso $(51.12 \mathrm{~g}$ a $55.27 \mathrm{~g}$ ) mientras que los del grupo DAR disminuyeron (49.01g a 44.15g). El grupo DAR mostró una disminución de triglicéridos, colesterol sérico total (de $125.761 \mathrm{mg} / \mathrm{dL}$ a $104.874 \mathrm{mg} / \mathrm{dL}$ ) y glucógeno muscular, pero un aumento de los triglicéridos en el músculo gastrocnemio en comparación con el grupo DAP (de $0.160 \mathrm{mg} / \mathrm{dL}$ a $0.259 \mathrm{mg} / \mathrm{dL}$ ). Estos resultados apuntan a una disminución de la velocidad de absorción de glucosa en el grupo de animales con una dieta con mayor cantidad de almidón resistente, la cual puede haber inducido una disminución en los procesos de síntesis de triglicéridos y colesterol conduciendo a un aumento del catabolismo de estos sustratos por el organismo, y adaptación plástica de los músculos para el uso de ácidos grasos en su metabolismo oxidativo.

Palabras clave: Almidón pregelatinizado; Dietas experimentales; Triglicéridos; Glucógeno; Colesterol.

\section{Introdução}

As modificações relacionadas ao estilo de vida e a dieta têm sido intimamente associadas ao crescente aumento de doenças crônicas não transmissíveis em todo o mundo, incluindo diabetes tipo 2 , hipertensão, dislipidemia, resistência à insulina, doenças cardiovasculares e obesidade. Com isso, a função dos alimentos e nutrientes na prevenção e tratamento, bem como nas complicações associadas a essas doenças tornou-se foco de preocupação de pesquisas nas últimas décadas (Si et al., 2017; Sun et al., 2018; Zheng et al., 2020; Medina Martinez et al., 2021).

As estratégias dietéticas vêm sofrendo modificações, como variações na composição dos macronutrientes, modificação do valor energético, inserção de ácidos graxos poliinsaturados, compostos bioativos, antioxidantes e fibra alimentar que inclui o amido resistente (AR). O AR foi definido pela EURESTA (European Flair Concertet Action on Resistant Starch) como a "quantidade total de amido e os produtos da degradação do amido que resistem à digestão no intestino delgado de indivíduos saudáveis" (Jiang \& Liu, 2002).

O principal interesse em relação ao AR é o seu papel fisiológico. Por não ser digerido no intestino delgado, este tipo de amido se torna disponível como substrato para fermentação pelas bactérias anaeróbicas do cólon (Englyst et al., 1992; Jenkins et al., 1998; Fuentes-Zaragoza et al., 2011). Os produtos resultantes dessa fermentação são os ácidos graxos de cadeia curta (AGCC), acético, propiônico e butírico, e gases como hidrogênio, dióxido de carbono e metano, dos quais cerca de $20 \%$ são excretados pela respiração (Topping \& Clifton, 2001). Dessa forma, o AR compartilha muitas das características e benefícios atribuídos à fibra alimentar no trato gastrintestinal (Fuentes-Zaragoza et al., 2011; Öztürk \& Mutlu, 2019).

Para propósitos nutricionais, o amido pode ser classificado como glicêmico ou resistente. O amido glicêmico é degradado a glicose por meio da ação das enzimas $\alpha$-amilase, glucoamilase e sacarase-isomaltase no intestino delgado, podendo ser classificado como amido rapidamente digerível (ARD), que é hidrolisado a glicose dentro de 20 minutos, ou amido lentamente digerível (ALD), que é convertido a glicose entre 20 e 110 minutos (Englyst et al., 1992). As diferenças na resposta glicêmica ao amido da dieta, está diretamente relacionada à taxa de digestão do amido, sendo que o ALD tem sido 
associado ao melhor controle da diabetes tipo 2 (Jenkins et al., 1998; Sun et al., 2018).

Há crescentes evidências de que alimentos com alto teor de AR podem contribuir para a regulação do metabolismo da glicose, melhorando as respostas glicêmicas e insulinêmicas (Harris, 2019), além de estarem associados a reduções nos níveis de colesterol e de triglicerídeos plasmáticos (Higgins et al., 2004), prevenção do câncer de cólon (Birt et al., 2013) e redução na ingestão de energia (Gentile et al., 2015), demonstrando efeito protetor contra o desenvolvimento da obesidade induzida pela dieta (Keenan et al., 2006; Maki et al., 2012).

Em razão dos efeitos fisiológicos e das contribuições positivas do amido resistente na dieta, esse trabalho teve como objetivo avaliar o efeito da substituição do amido pré-gelatinizado por amido resistente no metabolismo de lipídeos e de carboidratos em ratos machos Wistar.

\section{Metodologia}

\subsection{Material}

O amido de milho natural foi doado pela CornProducts®, Mogi-Guaçu, São Paulo, Brasil. O amido de milho resistente (HI-MAIZE® 260) foi doado pela National Starch Food Innovation®. A mistura de vitaminas e de sais minerais foi doada pela Agroceres®. Todos os outros ingredientes usados foram de pureza analítica.

\subsection{Animais}

Foram utilizados 20 ratos Wistar machos recém-desmamados, adquiridos do Biotério Central da UNICAMP/CEMIB. Os animais foram mantidos em gaiolas individuais, em ambiente com temperatura controlada $\left(21 \pm 1^{\circ} \mathrm{C}\right)$ e ciclo claro/escuro de 12 horas com acesso ad libitum a dieta e água $\mathrm{O}$ estudo foi aprovado pelo Comitê de Ética para Uso de Animais da Uniararas, Brasil, sob o protocolo $n^{\circ}$ 073/2005 e os animais utilizados neste estudo foram manuseados de acordo com os Princípios Éticos para Pesquisa Animal adotados pelo Colégio Brasileiro de Experimentação Animal.

\subsection{Caracterização físico-química dos amidos}

Os amidos foram analisados quanto a umidade, teor de proteína e de cinzas usando respectivamente os métodos $n^{\circ} 44-$ 15A, no 46-12 e n⿳0 08-01 da American Association of Cereal Chemists (AACC, 2000). Os lipídeos foram quantificados pelo método de Bligh \& Dyer (1959) e os carboidratos totais calculados pela diferença [100 - (\% proteína + \% lipídeo + \% umidade $+\%$ cinza )]. O teor de amido resistente foi determinando após a ração pronta utilizando o método de Goñi et al. (1996).

\subsection{Preparação das dietas}

$\mathrm{O}$ amido de milho foi cozido em autoclave, na proporção 1:8 (amido:água), por 30 minutos a $121^{\circ} \mathrm{C}$, resfriado e seco em estufa de circulação de ar a $60^{\circ} \mathrm{C}$, por 24 horas. Em seguida foi moído para obtenção da farinha de amido pré-gelatinizada desidratada. As dietas DAP (Dieta como amido pré-gelatinizado) e DAR (Dieta com amido resistente) (Tabela 1) foram preparadas pela mistura dos ingredientes com adição de água até formar uma liga, da qual foram preparados pequenos bolos que foram secos a $50^{\circ} \mathrm{C}$ por 16 horas. Após a preparação, a ração foi mantida refrigerada até o momento de ser oferecida aos animais. 
Tabela 1. Composição das duas dietas experimentais $(\mathrm{g} / \mathrm{kg})$.

\begin{tabular}{lcc}
\hline \multicolumn{1}{c}{ Ingredientes $(\mathrm{g})$} & \multicolumn{2}{c}{ Dietas } \\
\cline { 2 - 3 } & DAP* & DAR \\
\hline Amido pré - gelatinizado & 600 & 400 \\
Amido HI-Maize 260 & 0 & 200 \\
Celulose & 80,0 & 80,0 \\
Caseína & 200 & 200 \\
Sais minerais* & 35,0 & 35,0 \\
Vitaminas* & 15,0 & 15,0 \\
Óleo de Milho & 70 & 70,0 \\
Total & 1000 & 1000 \\
\hline
\end{tabular}

* Conforme descrito por Reeves et al. (1993). Fonte: Autores.

\subsection{Protocolo experimental}

Os animais foram alocados aleatoriamente em dois grupos $(\mathrm{n}=10)$ por 22 dias. O grupo DAP recebeu a dieta com 9,5\% de amido resistente e o grupo DAR a dieta com 18,1\% de amido resistente. Durante o experimento, monitorou-se o consumo de ração e de água. $O$ peso dos animais foi determinado no primeiro e no vigésimo segundo dia.

\subsection{Coleta de amostras}

No $22^{\circ}$ dia, os animais foram anestesiados com pentobarbital e eutanasiados. O sangue ( $\left.\pm 5 \mathrm{~mL}\right)$ coletado via punção cardíaca. Em seguida foi retirado o lóbulo direito do fígado e o músculo gastrocnêmio da perna direita, e uma porção do tecido foi alíquotada e congelada para dosagem de triglicerídeos e colesterol e outra porção foi imediatamente processada para a dosagem de glicogênio. Todo o procedimento foi feito sobre uma placa de alumínio previamente congelada $\left(-15^{\circ} \mathrm{C}\right)$. $\mathrm{O}$ sangue foi mantido em banho-maria a $37{ }^{\circ} \mathrm{C}$ por 20 minutos para coagulação e após realizado centrifugação a $3000 \mathrm{rpm}$ por 15 minutos, o soro foi dividido em alíquotas e congelado.

\subsection{Dosagem de glicogênio hepático e muscular}

Para quantificação da concentração de glicogênio muscular e hepático, foi usada a metodologia proposta por Lo et al. (1970). As amostras de fígado e músculo (50 mg de tecido fresco) foram incubadas em $500 \mu \mathrm{L}$ de $\mathrm{KOH} 30 \%$ saturado com $\mathrm{Na}_{2} \mathrm{SO}_{4}$ em banho-maria fervente até à dissolução total do tecido. As amostras foram resfriadas e precipitadas com $550 \mu \mathrm{L}$ de etanol e após 30 minutos em banho de gelo e depois centrifugadas a $3000 \mathrm{rpm}$ por 15 minutos. O precipitado foi dissolvido em $3 \mathrm{~mL}$ de $\mathrm{H}_{2} \mathrm{O}$ e retirado $1 \mathrm{~mL}$. Posteriormente, $1 \mathrm{~mL}$ de fenol $5 \%$ e em seguida $5 \mathrm{~mL} \mathrm{de} \mathrm{H}_{2} \mathrm{SO}_{4}$ concentrado foram adicionados a alíquota de $1 \mathrm{~mL}$ retirada. A leitura foi realizada em espectrofotômetro a $490 \mathrm{~nm}$ e para quantificação uma curva padrão de glicose foi elaborada.

\subsection{Dosagem de colesterol total e triglicerídeos}

A partir das amostras de sangue foram avaliados os níveis séricos de colesterol total (CT) (Colesterol Liquiform, Labtest Diagnóstica S.A., Brasil) e triglicerídeos (TG) (Triglicerídeos Liquiform, Labtest Diagnóstica S.A., Brasil), de acordo com o método descrito pelo fabricante. Os resultados foram expressos em $\mathrm{mg}_{\text {.dL }} \mathrm{de}^{-1}$ de plasma.

\subsection{Análise estatística}

Os resultados apresentados do consumo da ração e água foram analisados por análise de variância (ANOVA) e teste de Tukey $(\mathrm{p}<0,05)$. Os resultados das análises de triglicerídeos, colesterol total, glicogênio hepático e muscular foram apresentadas em gráficos do tipo "Box-plot” (McGill et al., 1978). 


\section{Resultados e Discussão}

A Tabela 2 demonstra que o amido de milho nativo e o amido resistente (HI Maize 260) usados para a produção das dietas experimentais apresentaram valores semelhantes em relação à composição centesimal de lipídeos e cinzas. Em relação a proteína, o maior valor foi encontrado no HI Maize 260.

Tabela 2. Composição centesimal do amido de milho nativo e do amido resistente comercial.

\begin{tabular}{ccc}
\hline Composição & Amido milho nativo $(\mathrm{g} / 100 \mathrm{~g})$ & HI Maize $260(\mathrm{~g} / 100 \mathrm{~g})$ \\
\hline Umidade & $10,70 \pm 0,20$ & $10,30 \pm 0,10$ \\
Proteína & $0,31 \pm 0,01$ & $0,58 \pm 0,01$ \\
Lipídeos & $0,48 \pm 0,01$ & $0,35 \pm 0,01$ \\
Cinzas & $0,25 \pm 0,01$ & $0,01 \pm 0,00$ \\
Carboidratos & $86,9 \pm 0,01$ & $85,9 \pm 0,01$ \\
Amido resistente & $14,7 \pm 0,50$ & $59,0 \pm 1,00$ \\
\hline
\end{tabular}

Médias \pm desvio padrão. Fonte: Autores.

A Tabela 3 apresenta o teor de amido resistente presente em cada uma das dietas formuladas.

Tabela 3. Teor de amido resistente das dietas.

\begin{tabular}{cc}
\hline Dietas & Amido resistente $(\%)$ \\
\hline DAP & 9,5 \\
DAR & 18,1 \\
\hline
\end{tabular}

Fonte: Autores.

O consumo de ração e água (Tabela 4) não apresentaram variações significativas entre os grupos de animais tratados com as dietas DAP e DAR, apresentando um consumo médio de ração e água de 3,64 g/dia e 12,35 mL/dia, respectivamente. Isso demonstra que a porcentagem de amido resistente da dieta não alterou a quantidade de ração e o volume de água consumidos pelos animais, assim como foi encontrado por Rech et al. (2014) onde a média de ingestão alimentar não diferiu entre os grupos experimentais de animais nas semanas de experimento. De maneira semelhante, Walter et al. (2005), ao analisarem os efeitos de dietas contendo AR em diferentes concentrações ( $0,3,9$ e $18 \%$ ), em um período experimental de 15 dias, também verificaram que a adição de AR às rações não influenciou no consumo entre os animais.

Tabela 4. Consumo de ração e de água dos ratos machos Wistar tratados com dietas contendo 9,5\% de AR (DAP) e de 18,1\% de AR (DAR), durante 22 dias.

\begin{tabular}{ccccc}
\hline \multirow{2}{*}{ Dia } & \multicolumn{2}{c}{${\text { Ração }(\mathrm{g} / \mathrm{dia})^{\mathrm{ns}}}$} & \multicolumn{2}{c}{ Água $(\mathrm{mL} / \mathrm{dia})^{\mathrm{ns}}$} \\
\cline { 2 - 5 } & DAP & DAR & DAP & DAR \\
\hline 2 & $1,6 \pm 0,5$ & $3,4 \pm 1,3$ & $12 \pm 6,3$ & $4,2 \pm 1,4$ \\
5 & $5,4 \pm 2,7$ & $3,3 \pm 1,1$ & $16 \pm 3,7$ & $7,2 \pm 5,4$ \\
7 & $4,0 \pm 1,8$ & $4,3 \pm 1,1$ & $16 \pm 4,3$ & $9,3 \pm 5,2$ \\
9 & $3,1 \pm 1,1$ & $3,0 \pm 0,6$ & $15 \pm 3,2$ & $13 \pm 5,3$ \\
12 & $4,8 \pm 2,3$ & $5,2 \pm 1,0$ & $14 \pm 5,2$ & $12 \pm 4,8$ \\
17 & $3,3 \pm 1,6$ & $2,4 \pm 0,5$ & $14 \pm 5,1$ & $11 \pm 7,4$ \\
19 & $3,9 \pm 1,6$ & $3,8 \pm 1,3$ & $19 \pm 9,3$ & $10 \pm 5,8$ \\
22 & $5,9 \pm 2,0$ & $4,2 \pm 1,7$ & $16 \pm 6,9$ \\
\hline
\end{tabular}

Médias \pm desvio padrão $(n=5)$. nd = não determinado. ${ }^{n s}$ não significativo ao nível de $5 \%$ de probabilidade pelo teste $F(p>0,05)$. Fonte: Autores. 
Em relação ao peso, os animais do grupo DAP apresentaram tendência a aumento de peso, enquanto os alimentados com a dieta DAR apresentaram diminuição do peso (Tabela 4). Como o produto absorvível proveniente do amido é a glicose, sua disponibilidade depende do acesso das enzimas amilases às ligações entre as moléculas de glicose que formam as cadeias do amido (amilose e amilopectina). As amilases produzem maltose, maltotriose, dextrinas e outros oligossacarídeos que são substratos de oligossacaridases e maltases, resultando em glicose (Murray et al., 1998). O AR e as fibras dietéticas podem reduzir a taxa de digestão e a atividade da enzima amilase prevenindo o aumento do nível de glicose, insulina e diminuindo a glicose plasmática pós-refeição (Carvalho et al., 2019). Como a maioria dos processos de síntese é induzida pela insulina, as diferenças no peso podem estar relacionadas às diferenças na insulinemia dos diferentes grupos (Petersen \& Shulman, 2018).

Figura 1. Peso inicial e final dos ratos machos Wistar tratados com dietas contendo 9,5\% de AR (DAP) e de 18,1\% de AR (DAR), durante 22 dias.

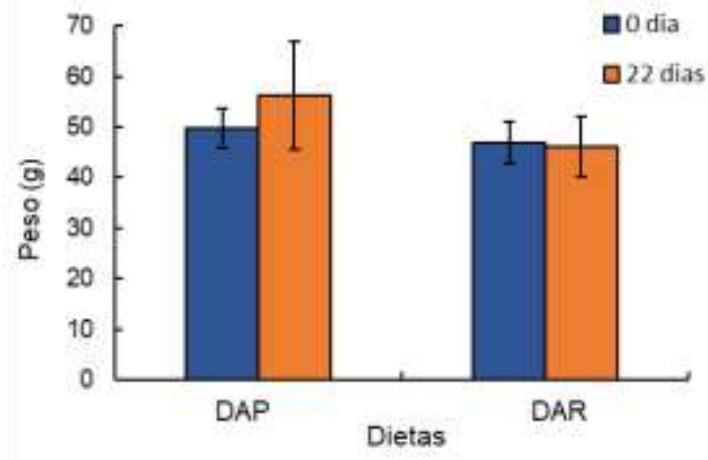

Fonte: Autores.

Os efeitos da dieta sobre os parâmetros bioquímicos estão apresentados na Figura 2. Os resultados obtidos para a concentração de colesterol total no soro (Figura 2A) mostraram que os animais que foram submetidos às dietas com maior quantidade de amido resistente (DAR) apresentaram menor concentração de colesterol (de 125,761 mg/dL para 104,874 $\mathrm{mg} / \mathrm{dL}$ ). Como a única variável na dieta foi o nível de AR, pode-se inferir que o aumento na percentagem de amido resistente levou à diminuição no colesterol total plasmático, possivelmente devido à diminuição de atividade da enzima HMG-CoA redutase que é a enzima controladora da síntese deste composto (Fonseca, 2005). Da mesma forma, Sun et al. (2018) relata que quando a dose de AR foi aumentada, os lipídeos hepáticos diminuíram significativamente, indicando efeitos hipolipidêmicos.

Em relação a concentração de triglicerídeos (Figura 2C) os resultados mostraram que os animais que consumiram a dieta DAR apresentaram menor concentração de triglicérideos no plasma, de $0,163 \mathrm{mg} / \mathrm{dL}$ para $0,114 \mathrm{mg} / \mathrm{dL}$ na dieta controle. Como a quantidade de lipídeos foi mantida constante em todas as dietas, as diferenças na concentração de triglicerídeos no plasma podem ser explicadas por alterações na sua síntese pelo fígado ou ainda sua absorção pelas células periféricas. O AR pode inibir o acúmulo de triglicerídeos no tecido adiposo, pois após a ingestão de AR, a velocidade de síntese de gordura é significativamente reduzida (Shen et al., 2015). Também, a razão insulina/glucagon deve estar mais baixa quando se aumenta a percentagem de amido resistente porque como a glicose é disponibilizada mais lentamente, o pico de insulina é menor (Monteiro \& Nascimento, 2013).

Para melhor entender o que estava acontecendo com o metabolismo de lipídeos foi analisado o comportamento da concentração de triglicérideos no músculo gastrocnêmio dos animais, visto que o músculo esquelético é um dos maiores consumidores de ácidos graxos e para isso possui uma reserva importante de triglicerídeos (Fontes, 2011). A Figura 2D mostrou que os ratos, que consumiram a dieta DAR, apresentaram tendência de aumento na concentração de triglicerídeos no 
músculo (de 0,160 mg/dL para 0,259 $\mathrm{mg} / \mathrm{dL}$ ), o que pode estar relacionado a porcentagem de amido resistente presente na dieta.

A concentração de triglicerídeos no musculo pode estar intimamente relacionada com os resultados obtidos para o glicogênio muscular descritos na Figura 2F. Como os animais estudados, no início do estudo eram recém desmamados, podese relacionar estes resultados com adaptações ocorridas nos indivíduos para se adaptarem a uma realidade de uma dieta com baixa concentração de glicose, ou seja, com baixa insulinemia. Provavelmente os animais submetidos às dietas com os amidos de maior resistência sofreram adaptações no sentido de expressão de fibras de maior capacidade para uso de ácidos graxos, ou seja, maior quantidade de fibras tipo I. Isto pode estar ocorrendo no músculo gastrocnêmio, por tratar-se de um músculo misto. Este tipo de adaptação ocorre principalmente induzido por treinamento físico aeróbio, mas também pode ocorrer por diminuição no suprimento de carboidratos (Huijing \& Jaspers, 2006).

Os resultados apresentados na Figura 2E mostraram que as dietas foram capazes de oferecer glicose suficiente para manter o glicogênio hepático, mostrando que os amidos disponibilizados de forma lenta foram suficientes para manter a glicemia, o que corrobora com o estudo de Prado-Silva et al. (2014) que não mostra diferença significativa para os parâmetros de glicogênio hepático e muscular nos ratos submetidos a dietas com diferentes concentrações de AR. A reserva de glicogênio hepático serve para manutenção da glicemia no jejum curto e sua ausência faz o fígado entrar em gliconeogênese usando como substrato, principalmente, os aminoácidos provenientes da degradação de proteínas musculares e albumina plasmática (Lehninger, 2005). Como a síntese de glicogênio quanto a gliconeogênese são reguladas, de forma inversa, pela razão insulina/glucagon (Monteiro \& Nascimento, 2013), os resultados mostraram que a possível diminuição nesta razão não induziu diminuição na concentração do glicogênio hepático. Relacionando com a obesidade esses resultados ocasionam muitos benefícios, pois a não diminuição no glicogênio hepático, evita que o organismo use as proteínas como fonte de glicose na gliconogênese e diminui a formação de corpos cetônicos quando tecidos utilizam outras fontes de energias, como oxidação de lipídeos, trazendo benefícios para diminuição de ácidos graxos nos tecidos adiposos.

Analisando a concentração de glicogênio muscular (Figura 2F) se observa que a dieta de maior conteúdo de amido resistente (DAR) promoveu menor concentração de glicogênio muscular (de 0,034 mg/g para 0,013 mg/g). A explicação para este resultado pode-se basear no fato de que o músculo gastrocnêmio é um músculo misto, composto de fibras tipo IIA e IIB. A concentração de glicogênio nestes dois tipos de fibras é diferente, as fibras IIB que são predominantemente glicolíticas possuem mais glicogênio que as do tipo IIA que são oxidativas. A plasticidade do músculo esquelético permite que ele se adapte, modificando sua constituição em fibras musculares, de acordo as necessidades do indivíduo. Esta adaptação ocorre principalmente como resposta ao treinamento físico (Costill \& Wilmore, 2001).

Trabalhos com animais depletados do glicogênio muscular por exercício físico mostram que a recuperação do glicogênio muscular é prioritária mesmo quando também há depleção do glicogênio hepático (Pereira, 2007) portanto não existe a possibilidade de haver uma economia na formação do glicogênio muscular induzida por concentrações mais baixas de glicose ou ainda por baixa taxa de insulina.

Pode-se inferir que nos animais usados no nosso experimento, a adição de AR, induziu modificações tanto no metabolismo de lipídeos quanto no de carboidratos. Estas modificações são consequência da diminuição da velocidade da absorção da glicose, provocada pelo efeito fibra induzido pelo amido resistente e/ou pelas lesões induzidas pelo aumento no teor de fibras ao trato intestinal dos animais. Deve ser destacar, que baseado principalmente nos dados obtidos com a dieta que contém maior concentração de amido resistente (DAR), propomos que houve adaptação no músculo gastrocnêmio com aumento no conteúdo de fibras IIA. Esta proposta baseia-se no aumento na concentração de triglicerídeos e na diminuição do glicogênio neste músculo, porém há necessidade de experimentos comprobatórios. 
Figura 2. Concentração de colesterol no soro (A); colesterol muscular (B); triglicerídeos no soro (C); triglicerídeos no musculo (D); glicogênio hepático (E) e glicogênio muscular (F) dos ratos machos Wistar tratados com dietas contendo 9,5\% de AR (DAP) e $18,1 \%$ de AR (DAR), durante 22 dias.
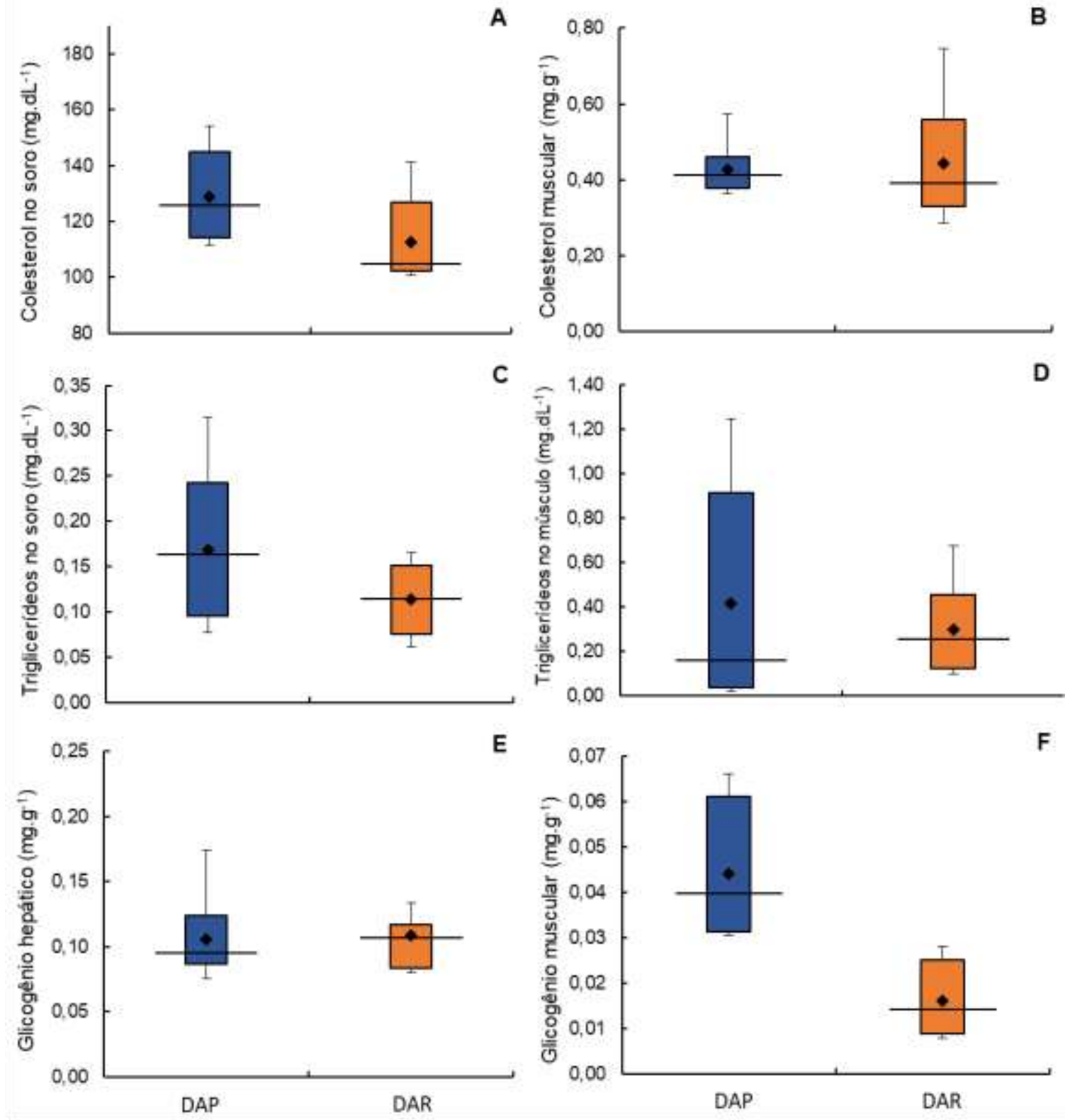

Fonte: Autores.

Baseados nos resultados desse estudo podemos inferir que a adição de amido resistente induziu modificações tanto no metabolismo de lipídios quanto no de carboidratos. Estas modificações são consequência da diminuição da velocidade da absorção da glicose, provocada pelo efeito fibra induzido pelo amido resistente e/ou pelas lesões induzidas pelo aumento no teor de fibras ao trato intestinal dos animais. Esta proposta baseia-se no aumento na concentração de triglicérides e na diminuição do glicogênio neste músculo, porém há necessidade de experimentos comprobatórios e que se estudem dietas com menor conteúdo de amido resistente. 


\section{Conclusão}

Este estudo mostrou que o aumento da concentração de AR na dieta pode levar a diminuição do peso e alterações nos metabolismos de lipídeos e carboidratos. A dieta com maior quantidade de AR demonstrou manter os níveis de glicogênio hepático, diminuir o glicogênio muscular, diminuir os lipídeos circulantes e aumentar a oxidação muscular dos ácidos graxos, levando à diminuição na síntese e aumento do catabolismo destes substratos pelo organismo.

\section{Agradecimentos}

Os autores agradecem à Coordenação de Aperfeiçoamento de Pessoal de Nível Superior (CAPES) pelo apoio financeiro (código 001), e ao Conselho Nacional de Desenvolvimento Científico e Tecnológico (CNPq) pela bolsa de doutorado de B. L. Tagliapietra (Processo 140805/2020-6).

\section{Referências}

AACC - American Association of Cereal Chemists (2000). Approved methods of the American Association of Cereal Chemists. Saint Paul, USA.

Birt, D. F., Boylston, T., Hendrich, S., Jane, J. L., Hollis, J., Li, L., McClelland, J., Moore, S., Phillips, G. J., Rowling, M., Schalinske, K., Scott, M. P. \& Whitley, E. M. (2013). Resistant starch: Promise for improving human health. Advances in Nutrition, 4, 587-601. https://doi.org/10.3945/an.113.004325.

Bligh, E. G. \& Dyer, W. J. (1959). A rapid method of total lipid extraction and purification. Canadian Journal of Biochemistry and Physiology, $37,911-917$.

Bloch, K. (1965). The biological synthesis of cholesterol. Science, 150, 19-28.

Carvalho, D. V., Silva, L. M. A., Alves Filho, E. G., Santos, F. A., De Lima, R. P., Viana, A. F. S. C, Nunes, P. I. G., Fonseca, S.G.D.C., De Melo, T. S., Viana, D. D. A., Gallão, M. I. \& De Brito, E. S. (2019). Cashew apple fiber prevents high fat diet-induced obesity in mice: an NMR metabolomic evaluation. Food \& Function, 10, 1671 - 1683. https://doi.org/10,1039 / c8fo01575a.

Costill, D. L. \& Wilmore, J. H. (2001) Fisiologia do esporte e do exercício. 1 ed. São Paulo, Brasil: Manole.

Englyst, H., Wiggins, H. S. \& Cummings, J. H. (1982). Determination of the non-starch polysaccharides in plant foods by gas-liquid chromatography of constituent sugars as alditol acetates. Analyst. 107, 307-318.

Fonseca, F. A. H. (2005). Farmacocinética das estatinas. Arquivos Brasileiros de Cardiologia, 85. https://doi.org/10.1590/S0066-782X2005002400003.

Fontes, R. (2011). Metabolismo do glicogênio. Universidade do Porto. Faculdade de Medicina do Porto, Porto: Portugal.

Fuentes-Zaragoza, E., Sánchez-Zapata, E., Sendra, E., Sayas, E., Navarro, C., Fernández-López, J. \& Pérez-Alvarez, J.A. (2011), Resistant starch as prebiotic: A review. Starch/Stärke, 63, 406-415. https://doi.org/10.1002/star.201000099.

Gentile, C. L., Ward, E., Holst, J. J., Astrup, A., Ormsbee, M. J., Connelly, S. \& Arciero, P. J. (2015). Resistant starch and protein intake enhances fat oxidation and feelings of fullness in lean and overweight/obese women. Nutrition Journal, 14, 1-10. http://purl.flvc.org/fsu/fd/FSU_pmch_26514213.

Goñi, I., Garcia-Diz, L., Mañas, E. \& Saura-Calixto, F. (1996). Analysis of resistant starch: a method for foods and food products. Food chemistry, 56, 445449 .

Harris, K. F. (2019). An introductory review of resistant starch type 2 from high-amylose cereal grains and its effect on glucose and insulin homeostasis. Nutrition Reviews, 77, 748-764. https://doi.or/10.1093/nutrit/nuz040.

Higgins, J. A., Higbee, D. R., Donahoo, W. T., Brown, I. L., Bell, M. L. \& Bessesen, D. H. (2004). Resistant starch consumption promotes lipid oxidation. Nutrition \& Metabolism, 6, 1-8.

Huijing, P. A. \& Jaspers, R. T. (2005). Adaptation of muscle size and myofascial force transmission: a review and some new experimental results. Scandinavian Journal of Medicine \& Science in Sports, 15, 349-80. https://doi.org/10.1111/j.1600-0838.2005.00457.x.

Jenkins, D. J. A., Wolever, T. M. S. \& Jenkins, A. L. (1988) Starchy foods and glycemic index. Diabetes care, 11, $149-59$.

Jiang, G. \& Liu, Q. (2002). Characterization of residues from partially hydrolyzed potato and high amylose corn starches by pancreatic $\alpha$-amylase. Starch Stärke, 54, 527-533. https://doi.org/10.1002/1521-379X(200211)54:11<527::AID-STAR527>3.0.CO;2-.

Keenan, M. J., Zhou, J., Hegsted, M., Pelkman, C., Durham, H. A., Coulon, D. B. \& Martim, R. J. (2015). Role of resistant starch in improving gut health, adiposity, and insulin resistance. Advances in Nutrition, 6, 198-205. https://doi.org/10.3945/an.114.007419

Lehninger, A. L., Nelson, D. L. \& Cox, M. M. (2005). Lehninger principles of biochemistry. 4 ed. New York, USA: W. H. Freeman and Company.

Lo, S., Russell, J. C. \& Taylor, A. W. (1970) Determination of glycogen in small tissue samples. Journal of Applied Physiology, $28,234-6$. 
Maæhlum, S., Hoøstmark, A. T. \& Hermansen, L. (1977). Synthesis of muscle glycogen during recovery after prolonged severe exercise in diabetic and nondiabetic subjects. Scandinavian Journal of Clinical \& Laboratory Investigation. 37, 309-16. https://doi.org/10.3109/00365517709092634.

Maki, K. C., Pelkman, C. L., Finocchiaro, E. T., Kelley, K. M., Lawless, A. L., Schild, A. L. \& Rains, T. M. (2012). Resistant Starch from High-Amylose Maize Increases Insulin Sensitivity in Overweight and Obese Men. Jounal Nutrition, 142, 717 - 723. https://doi.org/ 10.3945/jn.111.152975.

McGill, R., Tukey, J. W. \& Larsen, W. A. (1978). Variations of box plots. The American Statistician, 32, 12-16.

Medina Martinez, O. D., Vieira Theodoro, J. M., Grancieri, M., Lopes Toledo, R. C., Vieira Queiroz, V. A., Ribeiro de Barros, F. A., \& Duarte Martino, H. S. (2021). Dry heated whole sorghum flour (BRS 305) with high tannin and resistant starch improves glucose metabolism, modulates adiposity, and reduces liver steatosis and lipogenesis in Wistar rats fed with a high-fat high-fructose diet. Journal of Cereal Science, 99. https://doi.org/10.1016/j.jcs.2021.103201.

Monteiro, F. V. \& Nascimento, K. O. (2013). Associação do consumo do amido resistente na prevenção e tratamento do diabetes mellitus tipo 2. Revista Verde, $8,12-19$.

Murray, R. K., Granner, D. K., Mayes, P. A. \& Rodwell, V. W. (1998). Harper: Bioquímica. Um Livro Médico Lange. 8 ed. São Paulo, Brasil: Atheneu.

Öztürk, S., \& Mutlu, S. (2018). Physicochemical properties, modifications, and applications of resistant starches. In Starches for Food Application: Chemical, Technological and Health Properties (pp. 297-332). Elsevier. https://doi.org/10.1016/B978-0-12-809440-2.00008-3.

Pereira, K. D. (2007). Amido resistente, a última geração no controle de energia e digestão saudável. Ciência e Tecnologia em Alimentos, 27, 88-92. https://www.redalyc.org/articulo.oa?id=395940085016.

Petersen, M. C. \& Shulman, G. I. (2018). Mechanisms of Insulin Action and Insulin Resistance. Physiological Reviews, 98, 2033-2223. https://dx.doi.org/10.1152\%2Fphysrev.00063.2017.

Prado-Silva, L., Azevedo, L., Oliveira, J. A. C., Moreira, A. P. M., Schmiele, M., Chang, Y. K., Paula, F. B. A., \& Clerici, M. T. P. S. (2014). Sesame and resistant starch reduce the colon carcinogenesis and oxidative stress in 1,2-dimethylhydrazine-induced cancer in Wistar rats. Food Research International, 62, 609-617. https://doi.org/10.1016/j.foodres.2014.04.027.

Rech, C., Freygang, J., \& Azevedo, L. C. de. (2014). Efeito da farinha de banana verde sobre o perfil lipídico e glicídico de ratos Wistar. Alimentos e Nutrição, 25, $7-11$.

Reeves, P. G., Nielsen, F. H. \& Fahey, Jr. G. C. (1993). Committee Report AIN-93 Purified Diets for Laboratory Rodents: Final Report of the American Institute of Nutrition Ad Hoc Writing Committee on the Reformulation of the AIN-76A Rodent. The Journal of Nutrition, 123, 1939-1951. https://doi.org/10.1093/jn/123.11.1939.

Shen, L., Keenan, M. J., Raggio, A., Williams, C. \& Martin, R. J. (2011). Dietary-resistant starch improves maternal glycemic control in Goto-Kakizaki rat Molecular Nutrition \& Food Research, 55, 1499 - 1508. https://doi.or/10.1002/mnfr.201000605.

Si, X., Strappe, P., Blanchard, C., \& Zhou, Z. (2017). Enhanced anti-obesity effects of complex of resistant starch and chitosan in high fat diet fed rats. Carbohydrate Polymers, 157, 834-841. https://doi.org/10.1016/j.carbpol.2016.10.042.

Sun, H., Ma, X., Zhang, S., Zhao, D., \& Liu, X. (2018). Resistant starch produces antidiabetic effects by enhancing glucose metabolism and ameliorating pancreatic dysfunction in type 2 diabetic rats. International Journal of Biological Macromolecules, 110, 276-284. https://doi.org/10.1016/j.ijbiomac.2017.11.162.

Topping, D. L. \& Clifton, P. M. (2001). Short-chain fatty acids and human colonic function: roles of resistant starch and nonstarch polysaccharides. Physiological reviews, 81, 1031-1064

Walter, M., Silva, L. P. \& Perdomo, D. M. X. (2005). Biological response of rats to resistant starch. Revista Instituto Adolfo Lutz, $64,252-257$.

Zheng, B., Wang, T., Wang, H., Chen, L., \& Zhou, Z. (2020). Studies on nutritional intervention of rice starch- oleic acid complex (resistant starch type V) in rats fed by high-fat diet. Carbohydrate Polymers, 246, 116637. https://doi.org/10.1016/j.carbpol.2020.116637. 